\title{
GENETICS
}

\section{Genotype-phenotype correlations in patients with CPR deficiency}

Genetic mutations that underlie a deficiency in CPR (also known as P450 oxidoreductase) and result in a variant of congenital adrenal hyperplasia have been related to disease symptoms in a cohort analysis of patients with CPR deficiency.

"It became clear from studying increasing numbers of patients with CPR deficiency that the disorder has broad

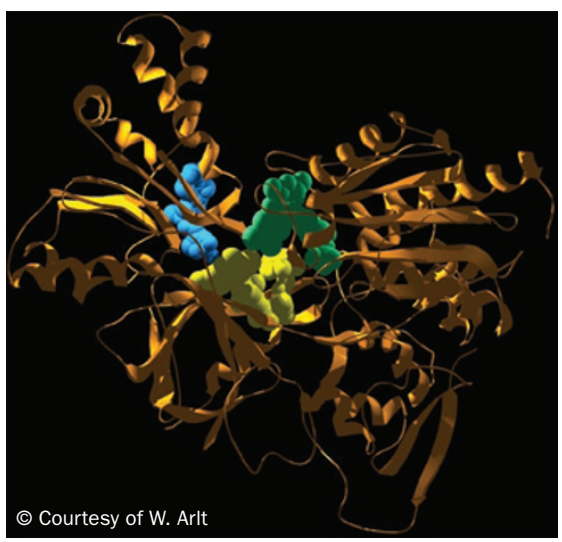

phenotypic variability," says Wiebke Arlt, senior investigator from the University of Birmingham, UK. "We brought together a cohort of 30 patients from 11 countries and carried out a detailed characterization study."

DNA from peripheral blood leukocytes was analyzed by direct sequencing of the POR gene, which encodes CPR. Partial or complete deletions or duplications were identified using multiplex ligationdependent probe amplification (MPLA). Krone et al. also devised a malformation score to measure symptoms associated with CPR deficiency, such as midface hypoplasia, synostosis of the cranium and large joints, and nonskeletal and pharyngeal malformations.

A number of mutations in the $P O R$ gene that remained undetected by direct sequencing were subsequently identified by MPLA, demonstrating the effectiveness of the assay for genetic analysis of CPR deficiency.
Several genotype-phenotype correlations were reported. Homozygosity for the most frequent amino acid substitution, Ala287Pro, was shown to predict moderate malformations in all patients and abnormal genital phenotype in girls but not in boys. Moreover, clusters of mutations were found to affect CPR protein structure, and urinary steroid metabolite profiling was shown to be useful as a noninvasive, comprehensive diagnostic method for CPR deficiency.

The researchers are now investigating the effects of POR mutations on drug metabolism, which could prevent steroid and drug overtreatment of patients with CPR deficiency.

\section{Andy McLarnon}

Original article Krone, N. et al. Genotype-phenotype analysis in congenital adrenal hyperplasia due to P450 oxidoreductase deficiency. J. Clin. Endocrin. Metab. doi:10.1210/jc.2011-0640 